Addendum to 'The NINJA-2 catalog of hybrid post-Newtonian/numerical-relativity waveforms for non-precessing black-hole binaries'

This content has been downloaded from IOPscience. Please scroll down to see the full text. 2013 Class. Quantum Grav. 30199401

(http://iopscience.iop.org/0264-9381/30/19/199401)

View the table of contents for this issue, or go to the journal homepage for more

Download details:

IP Address: 194.94.224.254

This content was downloaded on 23/01/2014 at 11:09

Please note that terms and conditions apply. 


\title{
Addendum to 'The NINJA-2 catalog of hybrid post-Newtonian/numerical-relativity waveforms for non-precessing black-hole binaries'
}

\author{
P Ajith ${ }^{1}$, Michael Boyle ${ }^{2}$, Duncan A Brown ${ }^{3}$, Bernd Brügmann ${ }^{4}$, \\ Luisa T Buchman $^{5}$, Laura Cadonati ${ }^{6}$, Manuela Campanelli ${ }^{7}$, \\ Tony $\mathrm{Chu}^{5,8}$, Zachariah B Etienne ${ }^{9}$, Stephen Fairhurst ${ }^{10}$, \\ Mark Hannam ${ }^{10}$, James Healy ${ }^{11}$, Ian Hinder ${ }^{12}$, Sascha Husa ${ }^{13}$, \\ Lawrence E Kidder ${ }^{2}$, Badri Krishnan ${ }^{14}$, Pablo Laguna ${ }^{11}$, \\ Yuk Tung Liu ${ }^{9}$, Lionel London ${ }^{11}$, Carlos Lousto $^{7}$, Geoffrey Lovelace ${ }^{2}$, \\ Ilana MacDonald ${ }^{8}$, Pedro Marronetti ${ }^{15}$, Satya Mohapatra ${ }^{6}$, \\ Philipp Mösta ${ }^{12}$, Doreen Müller ${ }^{4}$, Bruno C Mundim ${ }^{7}$, \\ Hiroyuki Nakano ${ }^{7}$, Frank Ohme ${ }^{12}$, Vasileios Paschalidis ${ }^{9}$, \\ Larne Pekowsky ${ }^{3,11}$, Denis Pollney ${ }^{13}$, Harald P Pfeiffer ${ }^{8}$, \\ Marcelo Ponce $^{7}$, Michael Pürrer ${ }^{16}$, George Reifenberger ${ }^{15}$, \\ Christian Reisswig ${ }^{5}$, Lucía Santamaría ${ }^{1}$, Mark A Scheel ${ }^{5}$, \\ Stuart L Shapiro ${ }^{9}$, Deirdre Shoemaker ${ }^{11}$, Carlos F Sopuerta ${ }^{17}$, \\ Ulrich Sperhake $^{5,17,18,19}$, Béla Szilágyi ${ }^{5}$, Nicholas W Taylor ${ }^{5}$, \\ Wolfgang Tichy ${ }^{15}$, Petr Tsatsin ${ }^{15}$ and Yosef Zlochower ${ }^{7}$ \\ ${ }^{1}$ LIGO_California Institute of Technology, Pasadena, CA 91125, USA \\ 2 Center for Radiophysics and Space Research, Cornell University, Ithaca, NY 14853, USA \\ ${ }^{3}$ Department of Physics, Syracuse University, Syracuse, NY 13254, USA \\ 4 Theoretisch Physikalisches Institut, Friedrich Schiller Universität, D-07743 Jena, Germany \\ 5 Theoretical Astrophysics 130-33, California Institute of Technology, Pasadena, CA 91125, USA \\ ${ }^{6}$ Department of Physics, University of Massachusetts, Amherst, MA 01003, USA \\ ${ }^{7}$ Center for Computational Relativity and Gravitation and School of Mathematical Sciences, \\ Rochester Institute of Technology, 85 Lomb Memorial Drive, Rochester, NY 14623, USA \\ ${ }^{8}$ Canadian Institute for Theoretical Astrophysics, University of Toronto, 60 St George Street, \\ Toronto, Ontario M5S 3H8, Canada \\ ${ }^{9}$ Department of Physics, University of Illinois at Urbana-Champaign, Urbana, IL 61801, USA \\ ${ }^{10}$ School of Physics and Astronomy, Cardiff University, The Parade, Cardiff, UK \\ ${ }^{11}$ Center for Relativistic Astrophysics and School of Physics, Georgia Institute of Technology, \\ Atlanta, GA 30332, USA \\ 12 Max-Planck-Institut für Gravitationsphysik, Albert-Einstein-Institut, Am Mühlenberg 1, \\ D-14476 Golm, Germany \\ ${ }^{13}$ Departament de Física, Universitat de les Illes Balears, Crta. Valldemossa km 7.5, \\ E-07122 Palma, Spain \\ ${ }^{14}$ Max-Planck-Institut für Gravitationsphysik, Albert-Einstein-Institut, Callinstraße 38, \\ D-30167 Hannover, Germany \\ 15 Department of Physics, Florida Atlantic University, Boca Raton, FL 33431, USA \\ ${ }^{16}$ Gravitational Physics, Faculty of Physics, University of Vienna, Boltzmanngasse 5, \\ A-1090 Vienna, Austria \\ ${ }^{17}$ Institut de Ciencies de l'Espai (CSIC-IEEC), Campus UAB, Bellaterra, E-08193 Barcelona, \\ Spain
}


18 Department of Physics and Astronomy, The University of Mississippi, University, MS 38677, USA

${ }^{19}$ CENTRA, Departamento de Física, Instituto Superior Técnico, Av Rovisco Pais 1, 1049-001 Lisboa, Portugal

Received 22 May 2013, in final form 24 May 2013

Published 18 September 2013

Online at stacks.iop.org/CQG/30/199401

\begin{abstract}
A recent paper (Ajith et al 2012 Class. Quantum Grav. 29 124001) described a catalog of 56 hybrid post-Newtonian/numerical-relativity waveforms modeling the inspiral, merger and ringdown of binary black hole systems spanning a range of mass ratios and spins. This catalog has been created and validated for use in the NINJA-2 project to study the sensitivity of gravitational-wave search and parameter-estimation algorithms. The contents of this catalog are being made available for public use. This addendum describes the public release.
\end{abstract}

PACS numbers: 04.25.Nx, 95.55.Ym, 04.80.Nn, 07.60.Ly, 04.25.D, 04.70.-s

S. Online supplementary data available from stacks.iop.org/CQG/30/199401/mmedia

The waveforms included in the supplementary data are released as part of the NINJA-2 project [1]. They may be freely used for any purpose but not redistributed. Please cite any usage as [3]. The catalog is divided into directories by submitting group, and each submission is described by a .bbh file, the format of which is described in [2]. This release includes only the $(\ell, m)=(2,2)$ modes, corresponding to the set validated in the paper.

\title{
References
}

[1] www.ninja-project.org/

[2] Brown D A et al 2007 Data formats for numerical relativity waves arXiv:0709.0093

[3] Ajith P et al 2012 Class. Quantum Grav. 29124001 